\title{
Le mot de la... Société francophone du diabète : Société francophone du diabète et Afrique francophone : une relation enrichissante à double sens
}

\section{French-speaking Africa and French-speaking Society of diabetes: A rewarding two-way relationship}

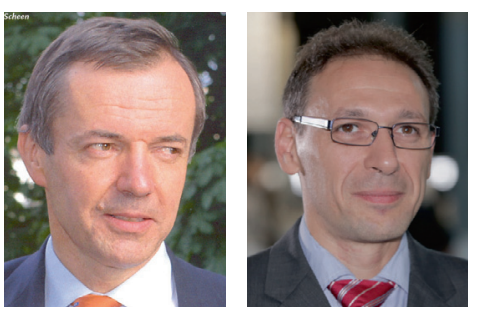

A.-J. Scheen ${ }^{1}$, S. Hadjadj ${ }^{2}$

${ }_{1}^{1}$ Président de la Société francophone du diabète.

${ }^{2}$ Secrétaire général de la Société francophone du diabète.

\section{Correspondance :}

Société francophone du diabète (SFD) Centre d'affaires Emergence Opéra

5, rue du Helder

75009 Paris

secretariat@sfdiabete.org

www.sfdiabete.org

() 2015 - Elsevier Masson SAS - Tous droits réservés.
- À l'initiative de son Directeur de la rédaction, Serge Halimi, la revue Médecine des maladies Métaboliques $(M m M)$ a décidé de publier un numéro spécial plus spécifiquement consacré à l'Afrique, et de faire coïncider sa sortie au moment du congrès annuel de la Société francophone du diabète (SFD), à Bordeaux (24-27 mars 2015). Nous nous réjouissons de cette heureuse initiative qui concrétise, en quelque sorte, l'intérêt réciproque des collègues africains pour la SFD, et de la SFD pour les collègues africains. Trop rares sont, en effet, les occasions de faire honneur aux travaux de l'Afrique dans le domaine de la diabétologie qui nous est cher. Relevons néanmoins, pour ceux qui sont intéressés, un supplément (Suppl.1) qui était paru en août 2013 dans la revue anglophone Diabetes Research \& Clinical Practice (journal de l'International diabetes federation [IDF/FID]), auquel plusieurs collègues et amis africains francophones avaient contribué.

- La revue officielle de la SFD est Diabetes \& Metabolism. Pour des raisons de concurrence à l'échelle internationale, Diabetes \& Metabolism a choisi de publier tous ses articles en anglais. Cette décision risquait, bien évidemment, de pénaliser le monde de la diabétologie francophone, pas toujours au fait de la langue de Shakespeare. Heureusement, MmM s'est rapidement révélée un remarquable outil de communication scientifique pour tous les médecins et paramédicaux francophones s'intéressant au diabète et aux maladies métaboliques. C'est particulièrement le cas pour toutes les personnes intéressées vivant dans l'Afrique francophone. Nous tenons à remercier Serge Halimi pour son implication considérable dans cette tâche, aussi importante qu'astreignante, et pour la qualité de cette revue qui représente un plus incontestable pour tous les membres de la SFD.

- Nous avons assisté, au cours des dernières années, à une augmentation constante de la participation des collègues de l'Afrique francophone au congrès annuel de la SFD. Au congrès de Montpellier de mars 2013, étaient présents 213 médecins venant d'Algérie, 86 du Maroc, 80 de Tunisie, 23 de Côte-d'Ivoire. Au dernier congrès, organisé à Paris, en mars 2014, ces chiffres étaient en nette progression. Nous avons dénombré 308 médecins algériens, 122 médecins marocains, 113 médecins tunisiens et 50 médecins provenant de l'Afrique sub-saharienne. Ainsi, les médecins de l'Afrique francophone représentaient plus de $15 \%$ de tous les participants. Et vous serez sans doute aussi nombreux au congrès de Bordeaux 2015 ! Cette large participation du Maghreb et, aussi, de l'Afrique sub-saharienne représente une véritable richesse pour notre société. En effet, le 
diabète sucré est une maladie universelle. Certes, il a un socle commun, quel que soit le pays, mais il présente aussi des particularités régionales évidentes et intéressantes à comparer. Partager les expériences des uns et des autres ne peut être que source d'enrichissement réciproque. Cette participation de l'Afrique francophone n'est pas seulement passive, mais elle devient aussi de plus en plus active, avec un nombre croisant de soumissions de résumés aboutissant à des présentations lors du congrès annuel de la SFD. Hélas, les contraintes logistiques ne permettent pas d'accepter tous les résumés soumis, et une sélection est opérée par un Conseil scientifique sur des critères objectifs appréciant la qualité et l'originalité. On ne peut que se réjouir de la qualité croissante des résumés soumis par nos collègues venant d'Afrique, ce qui témoigne du travail accompli au cours des dernières années.

- La SFD a, depuis 1985, une section paramédicale qui organise également, en parallèle du congrès des médecins, son congrès annuel. Cette section fête donc son trentième anniversaire cette année! Force est de constater que la participation des paramédicaux issus d'Afrique est encore limitée. Pourtant, les problèmes à résoudre sur le terrain sont nombreux et les besoins sont probablement énormes. La SFD Paramédicale, à l'initiative de sa présidente Jocelyne Bertoglio (voir son article dans ce même numéro), a organisé plusieurs ateliers de formation sur le territoire africain au cours des dernières années, en particulier en Algérie. Ceux-ci ont connu un grand succès et devront sans doute être répétés et étendus à l'avenir.

- De nombreux membres de la SFD sont régulièrement invités à donner des conférences sur le continent africain. II est évident que les congrès organisés sont d'une qualité croissante et rassemblent un nombre de plus en plus important de collègues. Épinglons, par exemple, le congrès maghrébin, celui de la Société marocaine d'endocrinologie, diabétologie et nutrition (SMEDIAN), ou encore les Journées de diabétologie d'Oran organisées de main de maître par notre collègue le Pr Mohamed Belhadj, et auxquelles participent régulièrement plusieurs membres de la SFD. La SFD souhaiterait, dans un proche avenir, étendre son partenariat avec les diverses sociétés savantes de diabétologie de l'Afrique francophone. Un des objectifs est d'organiser sur le sol africain une réunion officielle de la SFD, en étroite collaboration avec des organisateurs locaux. L'importance prise par le diabète et les maladies métaboliques en Afrique et l'intérêt des collègues africains francophones justifient certainement une telle initiative qui ne demande qu'à être concrétisée.

- Au cours de la dernière année, la SFD a considérablement modernisé, amélioré et enrichi son site web (www. sfdiabete.org), que nous vous invitons à consulter régulièrement (lire les informations complémentaires à ce sujet dans un autre article de S. Hadjadj, publié dans ce même numéro, et intitulé : «Société francophone du diabète (SFD) : nouvelles technologies et francophonie »). Sur le site de la SFD, vous pourrez trouver toutes les informations pertinentes relatives à la vie de notre société. Outre son congrès annuel du printemps, la SFD organise une journée consacrée à l'éducation thérapeutique en mai, une journée thématique en décembre, ainsi que la journée d'automne de la section paramédicale. Elle publie également des référentiels et coordonne de nombreux groupes de travail sur des thématiques spécifiques. Le site comprend également "Le coin de la bibliographie ", divers articles d'actualité, ainsi que des vidéos reprenant les conférences et présentations en langue française enregistrées lors des différentes activités de la SFD. Ces documents sont censés faciliter la diffusion des connaissances vers la francophonie, en général, et l'Afrique francophone, en particulier. Pour avoir accès à l'entièreté de ces informations, il convient d'être membre de la SFD. Vous trouverez les modalités pratiques d'inscription sur la partie du site accessible à tous. Si vous n'êtes pas encore membre de la société, n'hésitez pas à vous faire parrainer. Ainsi, les forces vives issues de l'Afrique occuperont une place de plus en plus importante au sein de la SFD.

- Le contenu de ce numéro de MmM consacré à l'Afrique francophone est à la fois riche et varié. Il comprend des articles à visée médico-économique, des articles consacrés à l'enseignement de la diabétologie, pour les médecins de première ligne et pour les spécialistes, en ce compris l'éducation thérapeutique, des articles dédiés à l'épidémiologie ou à des suivis de cohortes, des articles ciblant différentes situations particulières (diabète et grossesse, sujet âgé), des articles portant sur diverses approches thérapeutiques, ou encore des articles se focalisant sur une série de complications ou de pathologies associées.

Nous souhaitons une excellente lecture, intéressante, à tous ceux qui parcourront ce numéro remarquable de $\mathrm{MmM}$ consacré à la problématique diabétologique en Afrique francophone. La SFD se félicite du succès de ce numéro, remercie tous les participants, et congratule son Directeur de la rédaction et ses rédacteurs en chef.

\section{Déclaration d'intérêt}

Les auteurs déclarent n'avoir aucun conflit d'intérêt en lien avec la teneur de cet éditorial. 